\title{
Comparative Analysis of Black Swan Event Studies
}

\author{
Xinying LIU ${ }^{1} \quad$ Hang $\mathrm{LI}^{2} \quad$ Xiaobing $\mathrm{HU}^{2, *}$ \\ ${ }^{1}$ College of Electronic Information and Automation, Civil Aviation University of China, Tianjin 300300, China \\ ${ }^{2}$ The Flight Technology College, Civil Aviation University of China, Tianjin 300300, China \\ *Xiaobing HU. Email: huxb1975@163.com
}

\begin{abstract}
Black swan events have three basic characteristics: rarity, shock and post-event prediction. In recent years, black swan events occur frequently, which makes risk prevention and control in all sectors of society face severe challenges. Although scholars have done a lot of research on black swan events, there is no systematic summary. This paper mainly introduces the definition and classification of black swan in various fields. Due to the lack of a unified definition of black swan event, this paper classifies, summarizes and analyzes the definitions of various scholars with key words and research field classification points. At the same time, the research of domestic and foreign scholars on the prediction, impact and combination with risk management of black swan events is sorted out in order to provide useful reference for the study of black swan events.
\end{abstract}

Keywords: Black Swan events, Define classification, Risk management

\section{黑天鹅事件的研究对比分析}

\section{刘馨营 ${ }^{1}$ 李航 $^{2}$ 胡小兵 ${ }^{2, *}$}

\author{
1 中国民航大学飞行分校，天津 300300 \\ 2 中国民航大学 电子信息与自动化学院, 天津 300300 \\ *胡小兵.电子邮箱: huxb1975@163.com
}

摘要：黑天鹅事件具有稀有性，冲击性和事后预测性三个基本特征。近年来，黑天鹅事件频发，这使得社会各 领域的风险防控都面临严峻挑战。虽然学者们对黑天鹅事件进行大量研究, 但缺乏系统性的总结。本文主要围 绕黑天鹅在各个领域的定义分类进行介绍, 由于黑天鹅事件缺乏统一的定义, 因此文章以关键词和研究领域分 类点, 对各位学者的定义进行分类总结和分析。同时, 梳理了国内外学者关于黑天鹅事件的预测、影响、与风 险管理结合等方面的研究, 以期对黑天鹅事件的研究提供有益的参考。

关键字：黑天鹅事件，定义分类，风险管理

\section{1. 引言}

16 世纪以前, 所有人认为天鹅都是白色的, 对世 界的认知也是处于“天鹅是白色”的状态, 直到在澳大
利亚发现了第一只黑天鹅，才否定了这种坚定信念。 从此, 便用“黑天鹅”来喻指不可预测的重大稀有事 [1]。 现实世界中几乎各个领域都逃不过“黑天鹅”的影响。 2007 年美国次贷危机； 2008 年中国南方冰冻雪灾; 
2011 年福岛核电站泄漏; 2020 年新冠疫情。无论金 融、工业、公共卫生还是个人生活, 都逃不过它的控 制。这些事件摚乱了既定的秩序, 颠覆了人的惯性认 知, 改变了事件的发展。可以说“黑天我”事件影响深 远又无处不在。

\section{2. 黑天鹅事件定义的研究}

黑天我的概念最初是由拉丁诗人 Juvenal 提出的, 他写了《rara avis in terris nigroque simillima cygno》 (《地球上一种稀有的鸟类, 非常像一只黑天鹅》), Juvenal 的这句话在 16 世纪的伦敦是一个常见的表达 方式, 用来表达一些不可能的事情。近年来黑天我事 件在各个领域频发, 公众对于黑天鹅事件关注度也越 来越高。查阅相关文献发现, 众多学者透过黑天我事 件在逐步探索其本质。但在此过程中, 不难发现黑天 我事件的定义还不够明确, 不同的学者根据研究方向 不同使用了有区别的文字对黑天鹅的定义进行描述。

经济学领域中，金融专家纳西姆·尼古拉斯·塔勒 布在他的代表作《黑天鹅》一书中, 认为黑天鹅事件
需满足三个特征 ${ }^{[2]}$ :第一, 稀有性, 它在通常的预期之 外，过去没有任何能够确定它发生可能性的证据; 第 二, 冲击性，产生极端的影响;第三，事后预测性，尽 管它具有意外性, 但人的本性会促使我们在事后为它 的发生编制理由，似乎它是可以解释和预测的; Elisabeth Paté-Cornel[ ${ }^{[3]}$ 在研究黑天鹅事件与“完美风 暴”之间关系时，定义“黑天鹅”事件是用来描述不可 想象或极端不可能的事件。在公共安全领域, Terje Aven $^{[4]}$ 基于相关的风险观点, 认为黑天鹅事件是相对 于人的知识而言, 令人惊讶的极端事件。在政治领域 中, 周敏凯通过分析英国脱欧与美国特朗普当选两大 政治领域中的黑天我事件, 定义黑天鹅是出乎意料的 颠覆性事件。

由于黑天鹅事件缺乏统一的定义, 加之缺少覆盖 面足够广的资料, 近年来黑天鹅事件的主要研究局限 于某些固定领域。表 1 通过黑天鹅定义的关键词, 具 体列出各位学者对黑天鹅定义的探索结论, 表 2 是各 领域有代表性的定义总结, 用以体现各领域学者对定 义侧重点的选择。

表 1 黑天鹅事件定义关键词总结

\begin{tabular}{|c|c|c|}
\hline 关键词 & 定义内容 & 定义来源 \\
\hline $\begin{array}{c}\text { 稀有性+冲击性+事 } \\
\text { 后预测性 }\end{array}$ & 黑天鹅事件的特点:稀有性、冲击性、事后预测性。 & Taleb $^{[2]}, 2007$ \\
\hline 不可能 & 黑天鹅是用来描述不可想象或极端不可能。 & Elisabeth ${ }^{[3]}, 2011$ \\
\hline 令人惊讶+极端 & 相对于人的知识而言, 是令人惊讶的极端事件。 & Aven $T^{[4]}, 2012$ \\
\hline 不可预测 & 黑天鹅喻指不可预测。 & Masys A J ${ }^{[5]}, 2012$ \\
\hline 不可预测+重大 & 黑天鹅事件是不可预测的重大事件。 & 牟善军 $[6], 2013$ \\
\hline 不可能+存在性 & $\begin{array}{c}\text { 黑天鹅事件是极不可能发生实际却又发生并存在的事 } \\
\text { 件。 }\end{array}$ & 徐会永 ${ }^{[7]}, 2015$ \\
\hline 令人惊讶 & 对于观察者, 黑天鹅事件是令人惊讶的。 & Hajikazemi $^{[8]}, 2016$ \\
\hline $\begin{array}{c}\text { 异常值+抓住情绪+ } \\
\text { 广泛接受 }\end{array}$ & $\begin{array}{c}\text { 是一个异常值, 并且发生时, 应抓住人们的情绪并作 } \\
\text { 为重大事件被广泛接受。 }\end{array}$ & Mueller $\mathrm{J}^{[9]}, 2013$ \\
\hline $\begin{array}{l}\text { 意外性+极端影响+ } \\
\text { 突发 }\end{array}$ & 指满足意外性以及极端影响特点的突发事件。 & 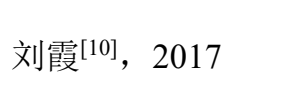 \\
\hline
\end{tabular}


表 2 不同领域黑天鹅定义总结

\begin{tabular}{|c|c|c|}
\hline 领域 & 定义 & 来源 \\
\hline \multirow{3}{*}{ 经济领域 } & $\begin{array}{c}\text { 非常难以预测, 且不寻常的事件, 它通常会引起市场 } \\
\text { 连锁负面反应甚至颠覆。 }\end{array}$ & 刘亮 ${ }^{[11]}$ \\
\hline & 极不可能发生, 实际又发生了的事件 & 茹瑜 ${ }^{[12]}$ \\
\hline & 用来描述造成广泛破坏的事件 & $\operatorname{David}^{\cdot[13]}$ \\
\hline 政治领域 & 出乎意料的颠覆性事件 & 周敏凯[14] \\
\hline \multirow[b]{2}{*}{ 公共安全领域 } & 意料之外的极端事件 & Aven $\mathrm{T}^{[4]}$ \\
\hline & $\begin{array}{c}\text { 当我们知道的和我们认为我们知道的之间的差距变得 } \\
\text { 很大 }\end{array}$ & Hajikazemia $S^{[8]}$ \\
\hline
\end{tabular}

\section{3. 黑天鹅事件研究内容}

黑天鹅无处不在:股市会突然崩盘;美国地产泡沫 会引发谁都没有预料到的次贷危机;一场突如其来的 大雪使得大半个中国陷入㿈瘠状态，带来上千亿元 的损失; 英国全民公投“脱欧”导致全球金融市场剧烈 震荡，外汇市场英镑汇兑闪电崩盘;特朗普击败希拉 里当选美国总统，这一超乎预计的事件导致黄金价 格先后经历暴涨和暴跌，其实我们每一天都被“黑天 我”环绕着。虽然其发生概率小, 预测难度大, 但一旦 发生都会在各个领域造成巨大冲击, 因此, 国内外学 者从黑天鹅事件的预测、影响、与风险管理结合等方 面对黑天鹅进行深入的研究。

\section{1 黑天鹅事件预测与识别}

通过查阅相关文献我们可以发现, 对于黑天鹅事 件的可预测性, 研究者们并没有达成共识。Taleb ${ }^{[2]}$ 认 为黑天鹅事件是不可预测的, 是高度不可能发生的事 件, 在我们的世界中黑天鹅事件是不可避免的。管理 这类事件的唯一方法是变强大, 从而对错误免疫, 免 受不良事件的影响。Green[ [15]在一定程度上支持了这 一观点, 他指出, 虽然这些类型的事件是不可预测的,
但相关组织应该准备好做出有效的反应, 并同时应对 不可想象的、规模惊人的事件所带来的心理冲击。

然而, 也有其他研究认为黑天鹅事件可以在一定 程度上被预测, Kenett ${ }^{[16]}$ 认为, 恰当的利用组织数据 可以帮助防止一些具有巨大破坏性的、意料之外的事 件。通过获取和合并数据, 以及构建数据驱动的风险 管理决策系统，改善现有的传统方法进而对意料之外 的风险进行预测; Murphy [17]将黑天鹅的概念应用于 化工过程风险评估, 他们认为黑天鹅事件有警示标志, 如果能够识别并防范小事件, 或许可以避开黑天鹅; Guntram Fritz ${ }^{[18]}$ 通过引入整体方法来更好地识别、评 估和管理大多数人认为是不可预测的“黑天鹅”事件; Dodson 和 Westney ${ }^{[19]}$ 指出重大事件可预测性丧失的 根本原因是缺乏识别战略风险的过程和治理。他们提 出了五个步骤来帮助识别和主动管理黑天鹅事件。 Kumar ${ }^{[20]}$ 指出目前对新冠疫情出现两种看法: 一方观 点认为新冠疫情为黑天鹅事件, 该事件具有巨大影响, 更难以预测，甚至更难以计算其可能性的特点; 相反 的观点是, 从所有角度来看, 这都是完全可以预料的 事件，因为所有风险驱动因素都在眼前。他结合两方 观点, 对当前印度的灾害管理进行分析, 并提出事件 给灾害风险管理系统及公共卫生系统带来的初步教 
训。

\section{2 黑天鹅事件影响研究}

Panagiotis Liargovas 和 Spyridon Repousis ${ }^{[21]}$ 选取 了 2001 2005 年间 9·11 事件，马德里火车爆炸，伦 敦火车爆炸三个事件, 利用事件分析法分析了此类 事件对希腊股市的冲击;Kunihiro Hanabusa ${ }^{[22]}$ 从石油 行业出发, 运用市场模型研究了 $9 \cdot 11$ 、伊拉克战争 对日本石油业的影响; David ${ }^{[13]}$ 通过列表、收集数据 分析了近年来气象灾难、恐怖袭击、传染病毒和地 质灾害等方面的黑天鹅事件对 GDP、10 年期债券和 2005 年至 2011 年 CBD 写字楼空置率等调查预测准 确性的影响。鄢梓遥等 ${ }^{[23]}$ 分析了 2020 年以来主要货 币汇率波动情况, 重点关注汇率风险对公司财务业绩 影响, 并且对加强公司规避汇率波动风险能力给出 初步建议。从认知黑天鹅事件入手, 结合航空、旅 游、交通、影视、公共卫生等行业的典型案例,分析 对企业经营的严重影响,探讨建立健全企业危机应对 机制。赵臻 ${ }^{[24]}$ 结合航空、旅游、交通、影视、公共 卫生等行业的典型案例,分析对企业经营的严重影响, 并对建立健全企业危机应对机制进行探讨。范春姣 [25]通过剖析李家沟煤矿" $1 \cdot 12$ "重大煤尘爆炸事故原 因, 得到煤矿风险管控环节中的缺陷和不足, 并且提 出将 PDCA 循环理论应用到煤矿风险管控保障措施 中, 以此来强化煤矿风险管控, 减少类似"黑天鹅"事件 的发生。江霞 ${ }^{[26]}$ 以在此次新冠疫情中“受益”的口罩 生产企业为例, 分析“黑天鹅”事件下“受益”企业的 财务风险, 并给出应对策略。

\section{3 黑天鹅在风险管理方面的研究}

杜利芳 ${ }^{[27]}$ 论述了黑天鹅与供应链及供应链风险 管理之间的关系, 并建立相应的风险评估体系对具体 案例进行分析, 提出相应风险控制策略; Aven $\mathrm{T}^{[28]}$ 将
黑天鹅事件分为未知的未知、已知的未知、已知的已 知三类进行分析, 并建立风险管理框架来降低黑天鹅 事件带来的冲击; 茹瑜[29]根据 2011 年 1 月到 2012 年 12 月期间遭受黑天鹅事件冲击的 32 家上市公司的财 务数据, 运用因子分析和 Logit 回归法对影响其股价 恢复能力的因素进行了分析; Posner ${ }^{[30]}$ 将经典的基础 研究方法与最新发展相结合, 引入了一个新的框架来 处理和评估极端风险, 展示了决策者如何才能最好地 应对这个时代的“黑天鹅”。赵志民 ${ }^{[31]}$ 在新冠疫情背 景下，从风险意识、组织体系、制度体系这三方面对 企业的风险管理进行举例分析并提出初步建议。莫静 金 ${ }^{[32]}$ 提出将"黑天鹅"事件的启示应用于医院护理危 机事件风险管理工作中,可提高医院护理人员护理危 机事件应急管理能力,降低护理危机事件发生率,提高 护理危机事件护理质量及护士职业认同感。

我们可以看出, 黑天鹅在社会的各领域中频繁发 生, 因此我们对于黑天鹅事件必须要有一个正确的认 识, 不能因为其发生概率小就将其忽略。人们喜欢在 事后用已有的知识和经验对事情进行分析和阐述, 但 这会影响人们对于事件本身的判断。所以虽然 $\mathrm{Taleb}^{[2]}$ 认为黑天鹅事件是不可预测的, 但很多事件发生之前 都有一定的警示标志, 虽然不一定会准确预测出事件 的发生及演变过程, 如果通过重视并防范一些基础风 险，或许可以避开黑天鹅事件或减少相应损失。

\section{4. 结论与展望}

总的来说, 近年来关于黑天鹅事件的研究得到了 长足的进步和发展，但也面临诸多的挑战。

（1）首先是关于黑天鹅事件的判定问题。现在 黑天鹅的研究多是拿来主义, 先称之为黑天鹅事件, 再对其进行研究, 关于一个事件如何判定为是否是黑 天鹅事件的研究还处于空白。 
（2）其次是黑天鹅事件的研究主要在但单一领 域开展，对于事件在领域之间扩散的广度、深度的问 题研究较少。

（3）最后, 在全球化的发展趋势下, 各个国家、 地区、各行业之间关系越来越紧密。如何防范黑天鹅 事件向全领域蔓延, 既要重视应急举措也要关注风险 管控。

\section{参考文献}

[1]金龙. “黑天鹅事件”对“中国公民出境游”带来的变 化[J]. 商,2015(24):28-29.SEEP

[2]Taleb, N.N. The Black Swan: The Impact of the Highly Improbable [M].New York: Random House Trade Paperbacks, 2007:3-4.

[3]Paté-Cornell, M.E. On black swans and perfect storms: risk analysis and management when statistics are not enough [J]. Risk Analysis,2012, 32 (11):1823-1833.

[4] Aven T. Risk, Surprises and Black Swans: Fundamental Ideas and Concepts in Risk Assessment and risk management [M].New York: Routledge, 2014:10-17. [5]Masys A J. Black swans to grey swans: revealing the uncertainty[J]. Disaster Prevention and Management: An International Journal, 2012, 21(3): 320-335.

[6]牟善军.当心黑天鹅事件一一浅谈风险评估的局限 性[J]. 安全、健康和环境, 2013,13(3):30,

[7]徐会永. 论科技期刊名称中“黑天鹅事件”的启示 [J]. 编辑学报,2015,27(1):16-18.

[8]Hajikazemi S, Ekambaram A, Andersen B, et al. The Black Swan-Knowing the unknown in projects [J]. Procedia-Social and Behavioral Sciences, 2016, 226:184-192.

[9]Mueller J , Stewart M G . The curse of the Black Swan[J]. Journal of Risk Research, 2013, 19:1-12.

[10]刘霞,白敏.中国股市 “黑天鹅”事件的形成演化机 理及其防控研究——中石化黄岛事件为例 $[\mathrm{J}]$. 安 阳工学院学报,2017,16(1):38-43 :
[11]刘亮.“黑天我”事件对资本市场的交易影响— 2012 年中国资本市场“黑天鹅”事件梳理[J].四川职业 技术学院学报,2013,23(02):17-20.

[12]茹瑜,许华,吴东生.黑天鹅事件冲击下上市公司股 价恢复能力研究——基于财务分析的视角 [J].金融经 济,2013(14):93-96.

[13] David M,Higgins. The black swan effect and the impact on Australian property forecasting $[\mathrm{J}]$. Journal of Financial Management of Property and Construction, 2013, 18(1):76-89.

[14]周敏凯.当前西方民主乱象的根源与未来走向一 一基于英美“黑天鹅事件”的理论思考 [J].人民论坛·学 术前沿,2017(13):32-40.

[15] Green, N. (2011), Keys to success in managing a black swan event [White paper], AON Corporation, Retrieved from http://www.aon.com/attachments/riskservices/Manage Black_Swan_Even_Whitepaper_31811.pdf [16] Kenett, R.S. (2013), Managing Risks with Data, Available at SSRN: http://ssrn.com/abstract $=2355474$ or http://dx.doi.org/10.2139/ssrn.2355474.

[17] Murphy, J.F. and Conner, J. (2012), Beware of the black swan: the limitations of risk analysis for predicting the extreme impact of rare process safety incidents, Process Safety Progress, 31(4), p. 330-333

[18]Werther, Albin G F . When Black Swans Aren।"t: On Better Recognition, Assessment, and Forecasting of Large Scale, Large Impact, and Rare Event Change[J]. Risk Management and Insurance Review, 2013, 16(1):123.

[19]Dodson, K. and Westney, R. (2014), Predictable projects in a world of black swans, Westney consulting group, Retrieved from http://www.westney.com/wpcontent/uploads/2014/05/Predictable-Projects-in-a-

World-of-Black-Swans.pdf.

[20]Mishra Pramod Kumar. COVID-19, Black Swan events and the future of disaster risk management in 
India[J]. Progress in Disaster Science,2020,8.

[21]Panagiotis Liargovas and Spyridon Repousis. The Impact of Terrorism on GreekBanks'Stocks: an event study[J]. International Research Journal of Finance and Economics, 2010.

[22]Kunihiro Hanabusa. Effects of foreign disasters on the petroleum industry in Japan: A financial market perspective[J]. Energy, 2010.

[22]社利芳. 基于黑天鹅理念的制造企业供应链风险 管理研究[D].太原理工大学,2014.

[23]滒梓遥, 邱晓, 朱英, 贾京坤. 双重“黑天鹅”事件下汇 率波动对石油公司财务业绩影响分析[J].当代石油石 化,2020,28(09):28-33.

[24]赵臻.基于黑天鹅事件背景下的企业危机管理能 力提升[J].兰州交通大学学报,2020,39(04):150-154.

[25]范春姣,黄继广, 马汉鹏.基于“黑天鹅”理念研究煤 矿风险管控措施[J].内蒙古煤炭经济,2019(19):79-80.

[26]江霞,赵青华.黑天鹅事件“受益”企业的财务风险 ——基于新冠肺炎疫情下口罩生产企业的反思 [J].企 业管理,2020(11):59-62.

[27]杜利芳. 基于黑天鹅理念的制造企业供应链风险 管理研究[D].太原理工大学,2014.

[28]Aven T. On the meaning of the black swan concept in a risk context [J]. Safety Science,2013, 57: 44-51.

[29]茹瑜, 许华, 吴东生. 黑天鹅事件冲击下上市公司股 价恢复能力研究——基于财务分析的视角 [J]. 金融经 济,2013(14):93-96.

[30] Posner K A. Stalking the Black Swan:Research and Decision Making in a World of Extreme Volatility[M]. Columbia University Press, 2010.

[31]赵志民. 从黑天鹅事件论卓越领导力与风险管理 图[J].中国质量,2020(04):10-14.

[32]莫静金,赖宜君,关小珊.基于 “黑天鹅”事件的启示 在医院护理危机事件风险管理中的应用分析 [J]. 临床 护理杂志,2016,15(01):2-5. 